\title{
The association between cognitive function and mortality pertained to specific but not general measures of cognitive function when health factors were considered
}

Smits CH, Deeg DJ, Kriegsman DM, et al. Cognitive functioning and health as determinants of mortality in an older population. Am J Epidemiol 1999 Nov 1;150:978-86.

QUESTIONS: In older people, does health influence the ability of cognitive functioning to predict mortality? Is mortality associated with general or specific measures of cognitive function?

\section{Design}

Population based cohort study of participants in the Longitudinal Aging Study Amsterdam with a mean 3.3 years of follow up.

Setting

11 municipalities in 3 culturally distinct geographic areas of the Netherlands.

\section{Participants}

2380 participants who were 55-85 years of age $(65 \%$ $\geqslant 65$ y of age, $51 \%$ women) in a random sample stratified by age and sex according to expected mortality after 5 years.

\section{Assessment of risk factors \\ General cognitive functioning (Mini-Mental State Exam- ination [MMSE]); information processing speed (Cod- ing Task adapted from the Alphabet Coding task); fluid intelligence (Raven's Coloured Progressive Matrices); learning and proportion retained (Auditory Verbal Learning Test); self rated health, functional limitations, medication use, and presence of chronic diseases; physi- cal performance (measures of mobility, balance, coordi- nation, and strength); and lung function were assessed at baseline (1992-3). Age, sex, educational level, and depressive symptoms (Center for Epidemiologic Studies Depression Scale [CES-D]) were also assessed.}

Source of funding: Netherlands Ministry of Health, Welfare, and Sports.

For correspondence: $\mathrm{Dr}$ C H Smits, Netherlands Institute of Mental

Health and Addiction,

Utrecht, Amsterdam, the Netherlands. Fax +31

(0)30297 1111.email: csmits@trimbos.nl

\section{Main outcome measure \\ Mortality status at August 1, 1996.}

\section{Main results}

All cognitive variables and all health variables (except for arthritis and other chronic diseases) were associated with increased mortality. After adjustment for age, sex, educational level, and depressive symptoms, all cognitive variables were still associated with increased mortality (table). When results were further adjusted for health

Associations between cognitive function and mortality at a mean follow up of 3.3 years

$$
\text { Cognitive measures }
$$

\begin{tabular}{lll} 
& Unadjusted for health & Adjusted for health \\
\hline General cognitive function & $0.88(0.78$ to 0.98$)$ & $0.9(0.8$ to 1.0$) \dagger$ \\
\hline Information processing speed & $0.7(0.6$ to 0.8$)$ & $0.9(0.7$ to 0.9$)$ \\
\hline Fluid intelligence & $0.8(0.7$ to 0.9$)$ & $0.86(0.75$ to 0.99$)$ \\
\hline Learning & $0.86(0.74$ to 0.99$)$ & $0.9(0.8$ to 1.1$) \dagger$ \\
\hline Proportion retained & $0.9(0.78$ to 0.98$)$ & $0.87(0.77$ to .97$)$ \\
\hline
\end{tabular}

${ }^{*}$ Adjusted for age, sex, education, and depressive symptoms.

†Not significant. factors, the associations between mortality and general cognitive functioning and learning were no longer statistically significant (table).

\section{Conclusions}

In older people, general cognitive functioning and specific cognitive measures (information processing speed, fluid intelligence, learning, and proportion retained) were associated with increased mortality. After adjustment for health factors, the associations between mortality and information processing speed, proportion retained, and fluid intelligence remained.

\section{COMMENTARY}

The ability to predict mortality in elderly people is clearly an important clinical and research activity. Similarly, determining the outcomes for people with cognitive dysfunction is relevant to those with this problem. The nature of the cognitive impairments most predictive of future mortality may also provide clues about the likely biological processes underlying this association. The study by Smits $e t$ al adds to the available evidence in support of the possibility that specific types of cognitive impairment in the elderly are predictive of increased mortality at 3 years. The biological basis for this association remains unclear and warrants further study.

This is a very powerful study with a large, population based sample and $>3$ years of follow up. This strength also suggests limitations of the study, such as the use of relatively brief and limited assessments of only a limited number of cognitive and health related variables. For example, the presence of dementia was not assessed, and dementia may be at least partially responsible for some of the associations found between cognitive dysfunction and mortality. It is noted, however, that the MMSE (which has some limited ability to screen for dementia) was shown to be a relatively weak independent predictor of mortality. Ultimately, the detected associations between specific types of cognitive dysfunction, when controlling for health variables measured, were relatively weak (ORs ranging from $0.8-0.9$ ). The strongest detected association was for non-participation in the study due to missing data (OR 0.39); non-participants were older, were less well educated, had more depressive symptoms, and were less cognitively and medically healthy.

In younger, better educated, and healthier seniors, the presence of slowed information processing or impairments in fluid intelligence and verbal recall all predict a slight increase in mortality at 3 years and should prompt an assessment for the cause of both the cognitive impairment and the increased risk.

Robert van Reekum, MD, FRCPC Baycrest Centre for Geriatric Care Toronto, Ontario, Canada 